\title{
Unilateral craniofacial microsomia
}

\author{
D. N. Upadhyaya, Vaishali Upadhyaya*, S. S. Sarkar* \\ Department of Plastic Surgery, King George Medical University, Lucknow, *Sarkar Diagnostics, C-1093, Sector-A, \\ Mahanagar, Lucknow, India
}

Correspondence: Dr. Vaishali Upadhyaya, Sarkar Diagnostics, C-1093, Sector - A, Mahanagar, Lucknow - 226006 , India.

E-mail:vshali77@indiatimes.com

An 18-years old young man who had a malformed right external ear since birth (microtia) presented to a plastic surgeon for treatment. He also had diminished hearing on the right side.

Examination revealed a microtic right pinna. The ear was grossly malformed and hypoplastic and the external auditory meatus were absent (Meurman Type II). Along with the ear deformity, he also had conductive deafness on the same side. His other examination findings included retrognathia, microgenia, deviation of the lower jaw midline to the right, occlusal cant to the right and a generalized mild hypoplasia of the entire right side of the face. The malformed ear on the right was set lower than the normal ear on the left.

The facial nerve was however spared. There were no problems with opening the mouth and there seemed to be no apparent involvement of the orbits or any bones other than the mandible. There was no malocclusion. Based on these findings, he was diagnosed to have unilateral craniofacial microsomia.

He was subsequently referred to us for a three-dimensional CT scan of the face.

CT was performed on a six-slice scanner (Emotion Six, Siemens, Erlangen, Germany). Three-dimensional reconstructions were done. CT revealed a grossly hypoplastic ramus of the mandible on the right side with vertical height discrepancy from the normal left side, a deformed right mandibular condyle and disturbed relation of the condyle to the glenoid fossa of the temporo-mandibular joint (TM joint), a discontinuity of the zygomatic process, a cant of the occlusal plane to the right, absent external auditory canal, absent middle ear and absence of pneumatization of the mastoid air cells [Figures 1-5]. The internal ear appeared normal. The maxillary height on the affected side was also decreased and there was a cant to the maxillary arch to the right side. The occlusal relationships between the arches were, however, maintained. Other unrelated findings included a polyp in the left maxillary antrum and mucosal thickening in the right antrum.
The patient was explained the spectrum of problems and the plastic surgeon advised a distraction lengthening of the ramus of the mandible on the right side with orthodontia and staged ear reconstruction to which he consented.

\section{Discussion}

Craniofacial microsomia is a syndrome in which facial growth is affected unilaterally or bilaterally. This syndrome has been known by various other names such as the "first and second branchial arch syndrome", "otomandibular dysostosis", "oculoauriculovertebral sequence", "Goldenhar syndrome", "lateral facial dysplasia" and "hemifacial microsomia". The condition may vary from mild to severe. Commonly, the ear oral cavity and mandible are involved.

Theories of mesodermal deficiency and vascular defects of the stapedial artery have been proposed..$^{[1]}$

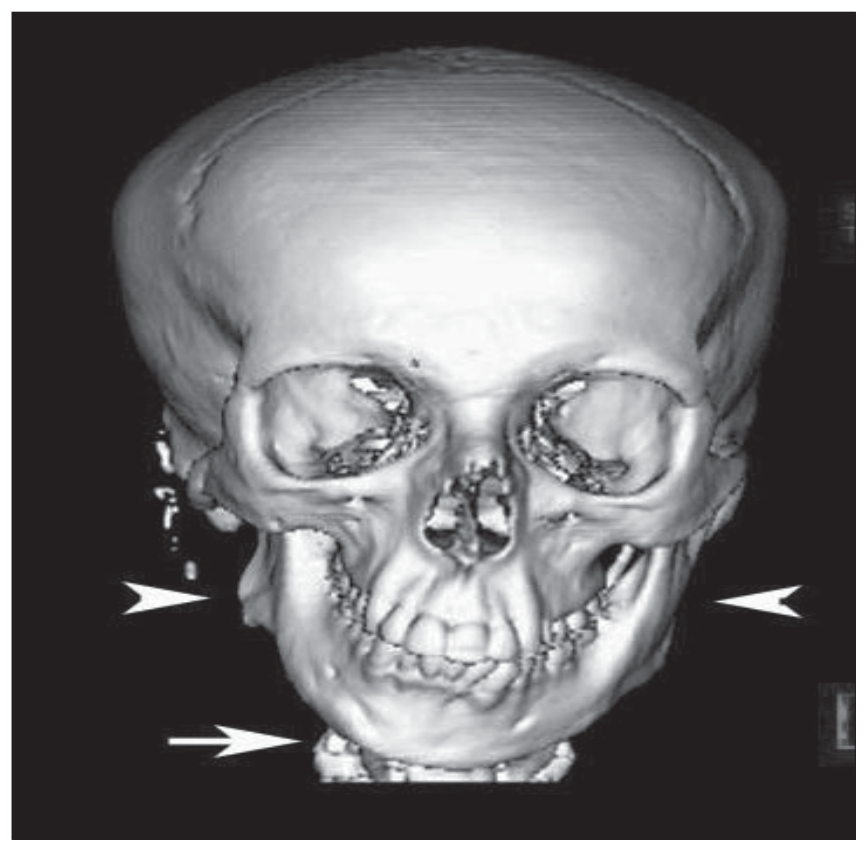

Figure 1: 3D reconstruction face (Frontal view) showing asymmetry in bilateral rami, hypoplastic right ramus, canted occlusal plane to the right, deviation of the chin to the right and short maxillary height on the right side 


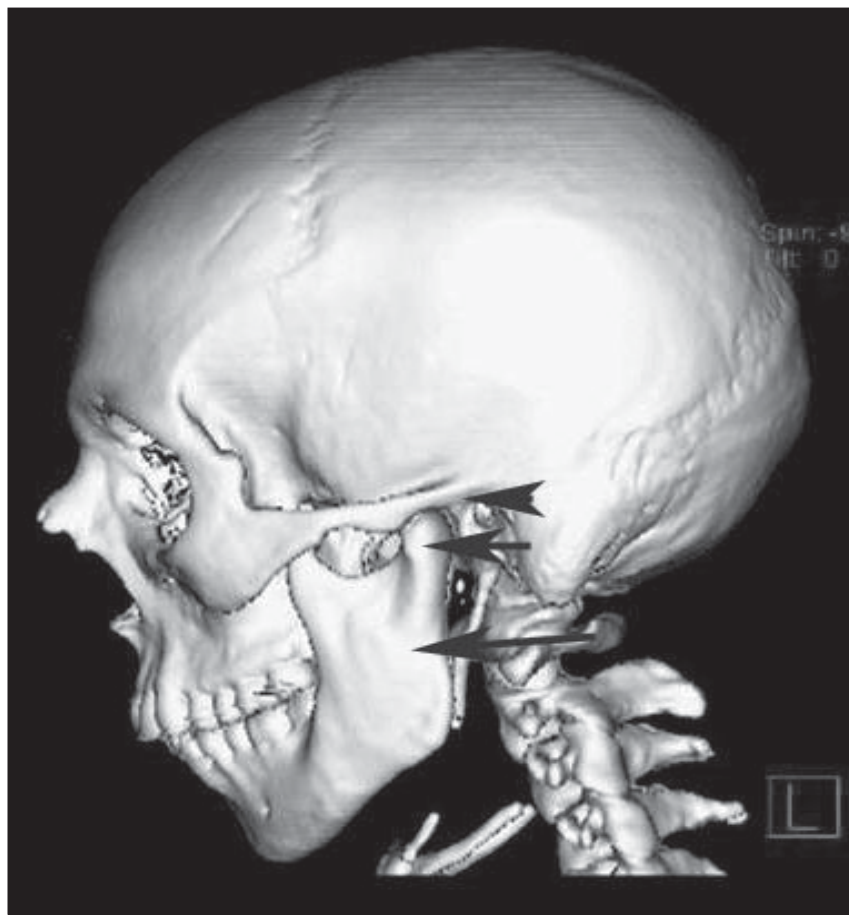

Figure 2: 3D reconstruction face (Left lateral view) showing normal ramal height, normal shaped condyle, normal condyle-glenoid fossa relationship and normal zygomatic arch

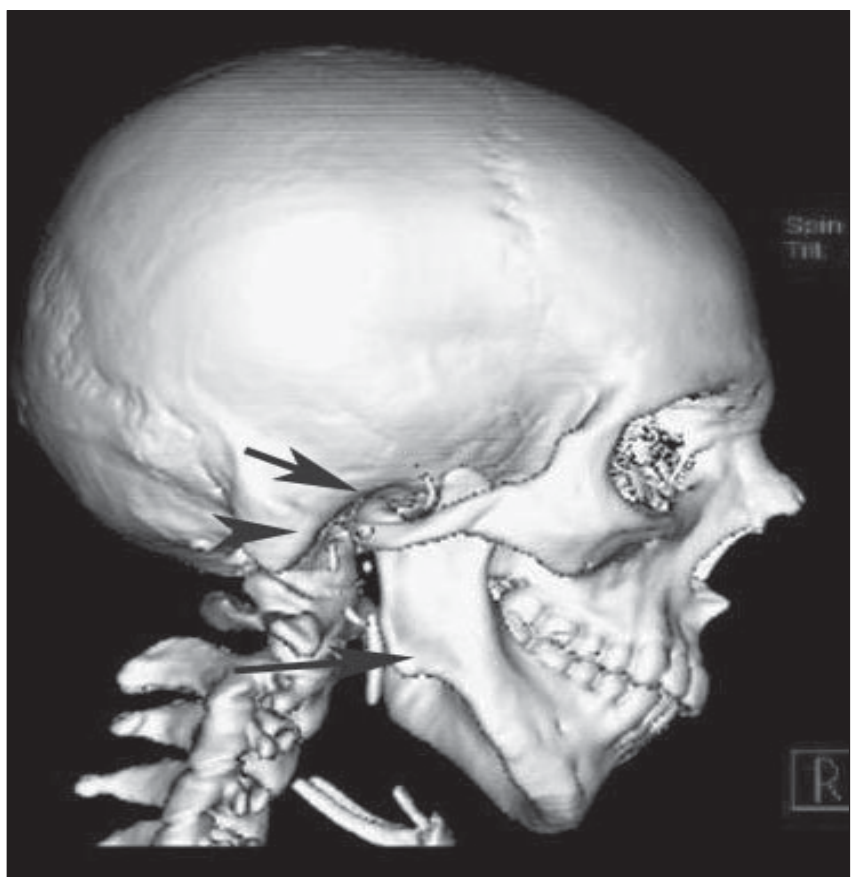

Figure 3: 3D reconstruction face (Right lateral view) showing abnormal ramal height, deformed condyle, disturbed relation of the condyle to the glenoid fossa and discontinuity of the zygomatic arch

The initial radiologic investigation is usually a lateral cephalogram. It provides information on maxillo-mandibular relationships and demonstrates the deviation from normal of the bone and soft tissue profiles. Postero-anterior and basilar cephalograms show the facial midline and degree

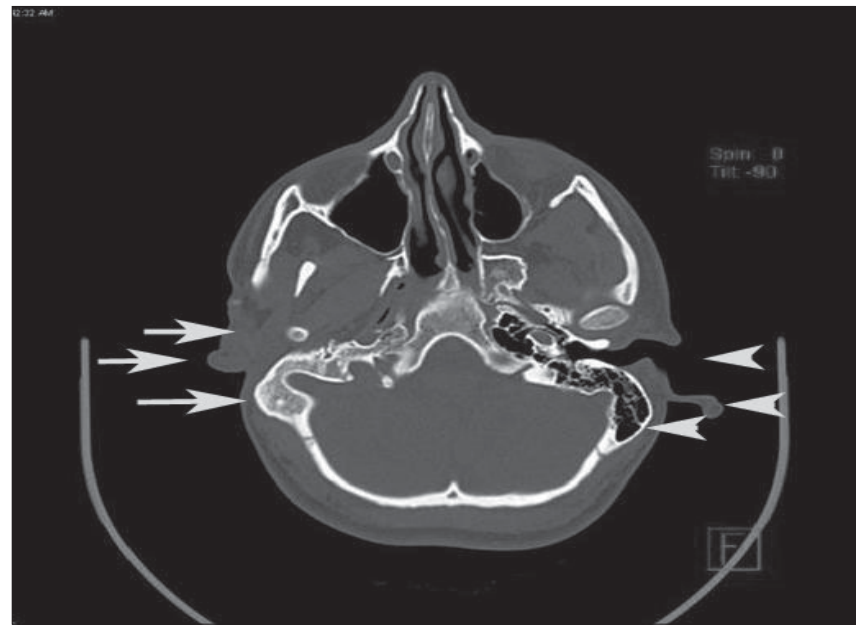

Figure 4: Axial section showing normally formed pinna and external auditory canal on the left side with well pneumatised mastoid bone. Right side shows malformed and displaced external ear, absent external auditory canal and loss of pneumatisation of the mastoid air cells

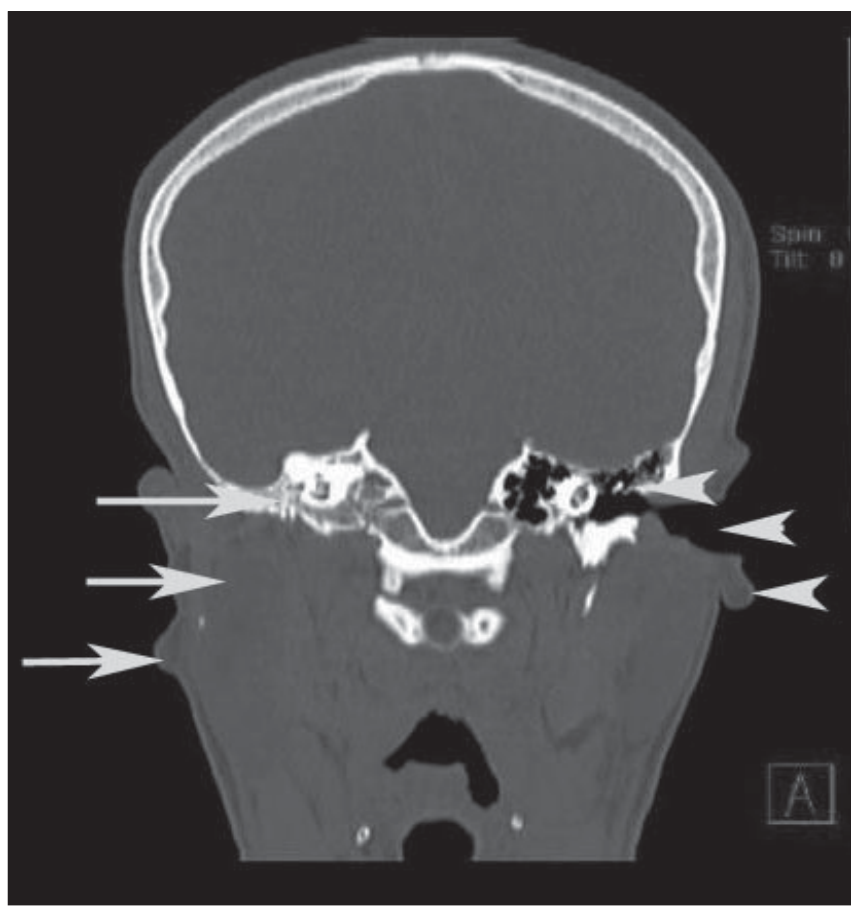

Figure 5: Coronal section showing normally formed pinna, external auditory canal and middle ear on the left side with well pneumatised mastoid bone. Right side shows malformed and displaced external ear, absent external auditory canal and middle ear with loss of pneumatisation of the mastoid air cells

of facial asymmetry. Orthopantomograms (OPGs) are also helpful as they allow comparison of mandibular rami and condyles. Lateral and PA cepahalograms and OPGs help the surgeon in planning surgery and give an idea of the amount of lengthening required or the degree of advancement needed.

The most important investigation these days, however, is the CT scan as it provides detailed information on bone and soft 
tissue asymmetry. It has become the fundamental diagnostic and evaluation tool for all patients with craniofacial microsomia. ${ }^{[2]}$ Three-dimensional reconstruction provides excellent skeletal visualization and images can be analyzed from any angle. Baseline measurements can be done which can be later compared with post-operative studies.

CT can help us identify the three major features of craniofacial microsomia, which include auricular, mandibular and maxillary hypoplasia. The most obvious skeletal deformity is the mandible. The ascending ramus can be absent or reduced in height and is usually displaced toward the midline. The chin is deviated towards the affected side. The mandibular condyle may be hypoplastic and malformed. The TM joint deformity can range from mild hypoplasia of the condyle with normal joint anatomy to a grossly disorganized joint anatomy and pseudo-articulation of the condyle at the cranial base. The maxilla is also reduced in height and eruption of mandible and maxillary molars is delayed. The zygomatic arch may be decreased in length or absent.

The mastoid process can be hypoplastic with partial or complete lack of pneumatization of the mastoid air cells. The styloid process may be short or absent. The orbit maybe reduced in dimensions and frontal bone can be flattened. There can also be malformations of cervical vertebrae, such as hemivertebrae, vertebral fusion and basilar impression.

Extraskeletal anomalies can also be identified. These include hypoplasia of muscles of mastication, small malformed or absent auricles, atresia of the external auditory canal and hypoplasia or aplasia of the parotid gland.

Cranial CT scan can reveal hypoplasia of cerebrum and corpus callosum as well as hydrocephalus.

Goldenhar syndrome, which is considered a variant of craniofacial microsomia, is differentiated by the presence of ocular abnormalities such as lipoma, lipodermoid, epibulbar dermoid or coloboma and cervical spine anomalies.

Many classification systems have been described for craniofacial microsomia. A classification which graded mandibular deficiency was given by Pruzansky in 1969.[3]
Grade I- minimal hypoplasia of the mandible, Grade IIfunctioning but deformed TM joint with anteriorly and medially displaced condyle and Grade III- absence of the ramus and glenoid fossa.

This was later modified by Kaban, Padwa and Mulliken in 1998. ${ }^{[4]}$ They subdivided Type II into A and B. In Type II A, the mandibular ramus, condyle and TM joint are present but hypoplastic and abnormal in shape. In Type II B, the mandibular ramus is hypoplastic and markedly abnormal in form and location. There is no articulation with the temporal bone.

Vento, LaBrie and Mulliken in $1991^{[5]}$ gave the OMENS classification of craniofacial microsomia. It designates each of the five major areas of involvement in craniofacial microsomia; O-orbital, M-mandibular, E-ear, N-facial nerve and S-soft tissue.

The images in this report beautifully demonstrate CT findings in patients with craniofacial microsomia. A detailed and accurate description by the radiologist of the many skeletal and extra-skeletal anomalies found in patients of craniofacial microsomia can help the surgeon plan the best possible approach to improve not just the patient's appearance but also go a long way in improving his quality of life.

\section{References}

1. McCarthy JG, Grayson BH, Coccaro PJ, Wood-Smith D. Craniofacial microsomia. In: McCarthy JG, editor. Plastic Surgery, $1^{\text {st }}$ ed. WB Saunders: Philadelphia; 1990. p. 3054-100.

2. McCarthy JG, Hopper RA, Grayson BH. Craniofacial microsomia. In: Mathes SJ, Hentz VR, editor. Plastic surgery. $2^{\text {nd }}$ ed. Elsevier Saunders: Philadelphia; 2005. p. 113-34.

3. Pruzansky S. Not all dwarfed mandibles are alike. Birth Defects 1969;5:120.

4. Kaban LB, Padwa BL, Mulliken JB. Surgical correction of mandibular hypoplasia in hemifacial microsomia: The case for treatment in early childhood. J Oral Maxillofac Surg 1998;56:628-38.

5. Vento AR, LaBrie RA, Mulliken JB. The O.M.E.N.S. classification of hemifacial microsomia. Cleft palate Craniofac J 1991;28:68-77.

Source of Support: Nil, Conflict of Interest: None declared. 